NASA Technical Memorandum 106465

AIAA-94-0139

\title{
Refraction of High Frequency Noise in an Arbitrary Jet Flow
}

Abbas Khavaran

Sverdrup Technology, Inc.

Lewis Research Center Group

Brook Park, Ohio

and

Eugene A. Krejsa

National Aeronautics and Space Administration

Lewis Research Center

Cleveland, Ohio

Prepared for the

32nd Aerospace Sciences Meeting and Exhibit

sponsored by the American Institute of Aeronautics and Astronautics

Reno, Nevada, January 10-13, 1994 



\title{
Refraction of High Frequency Noise in an Arbitrary Jet Flow
}

\author{
Abbas Khavaran" \\ Sverdrup Technology, Inc. \\ Lewis Research Center Group \\ Brook Park, Ohio 44142 \\ and \\ Eugene A. Krejsa ${ }^{\dagger}$ \\ National Aeronautics and Space Administration \\ Lewis Research Center \\ Cleveland, Ohio 44135
}

\begin{abstract}
Refraction of high frequency noise by mean flow gradients in a jet is studied using the ray-tracing methods of geometrical acoustics. Both the two-dimensional (2D) and three-dimensional (3D) formulations are considered. In the former case, the mean flow is assumed parallel and the governing propagation equations are described by a system of four first order ordinary differential equations. The 3D formulation, on the other hand, accounts for the jet spreading as well as the axial flow development. In this case a system of six first order differential equations are solved to trace a ray from its source location to an observer in the far field. For subsonic jets with a small spreading angle both methods lead to similar results outside the zone of silence. However, with increasing jet speed the two prediction models diverge to the point where the parallel flow assumption is no longer justified. The Doppler factor of supersonic jets as influenced by the refraction effects is discussed and compared with the conventional modified Doppler factor.
\end{abstract}

\section{Introduction}

Propagation and refraction of sound emitted from convecting multipole sources embedded in a shear flow has its application in jet noise studies. The shrouding effect of the mean flow and its contribution to the directivity of jet noise has been studied by numerous researchers in the past. It is well known that the operator part of Lilley's ${ }^{1}$ equation contains the effect of refraction as well as the source convection, with the right hand side of the equation representing, at least to a large degree, the source term only. For axisymmetric jets, this equation can conveniently be written in cylindrical coordinates and with appropriate transformations a turning point problem is formulated.

* Senior Research Engineer, Member AIAA.

$\uparrow$ Deputy Branch Chief, Propeller and Acoustic Technology Branch, Member AIAA.
Thus, $\mathrm{Mani}^{2,3}$ studied the mean flow interaction of round jets at subsonic eddy convection velocities for plug-flow jets. The source term in his model is a quadrupole convecting along the center-axis of the jet. He further argues that the field of sources located symmetrically about the jet axis is well represented by that of one source on the axis. With these assumptions Mani avoided considerable mathematical difficulty and obtained good agreement with data for both cold and hot jets except at high frequencies.

The high frequency solution is believed to provide a reasonably good approximation to the refraction problem even at Helmholtz numbers as small as on $e^{4,5,6}$, and is of particular interest in the prediction of the noise directivity of high speed jets. A high frequency asymptotic solution for arbitrarily located convected singularities in an axisymmetric parallel flow was derived by $\mathrm{Balsa}^{4}$. In his model, the turbulent properties of the jet and its acoustic radiation are coupled through a directivity factor for each of the quadrupoles contained within the turbulent eddy.

To study the radiation pattern of non-axisymmetric jets, Goldstein ${ }^{7}$ derives a circumferential directivity factor in the high frequency limit. For an inviscid parallel flow, he obtains a Green's function for a convecting monopole source when the Mach number and temperature are arbitrary functions of the cross-flow coordinates. The rays of geometrical acoustics (GA) are traced by following the projection of each ray on a cross-stream plane. Following Goldstein's work, a high frequency Green's function for convecting multipole sources in a spreading jet was developed by Durbin ${ }^{B}$ and used to predict the noise directivity of quadrupole sources convecting along the centerline of an axisymmetric jet. It is generally accepted that the high frequency acoustic sources lie in the neighbourhood of the shear layer development, which for an axisymmetric jet is simply a ring about the jet axis. However, by studying an on-axis source point, Durbin ${ }^{9}$ was able to simplify the directivity factor significantly and show some important features of the refraction problem.

Implicit in the use of the high frequency approximation is the assumption that the acoustic wavelength is shorter than the characteristic length of the mean flow. In addition, if turbulence length scale is small relative to 
the wavelength at which noise is produced, Lilley's equation suggests that the sound field is the result of radiation by independent correlation volume elements within the jet, each moving with the local convection velocity. A convecting ring source problem was done in ${ }^{10}$ by first considering an off-axis point source, leading to a 3D rayacoustic problem, and then carrying out a circumferential integration for an axisymmetric jet. In addition, the mean flow velocity profile was modeled to account for the streamwise development of the jet from a top-hat, near the exit, to a Gaussian, representative of the downstream fully developed flow. The size of zone of silence was found to be sensitive to jet spreading angle as well as the mean flow gradients surrounding any source point. Further, it was shown that the Doppler factor is influenced by the refraction effects and varies from point to point on a ring source.

The purpose of the present paper is to further our understanding of the refraction of high frequency jet noise and its application to jets numerically computed from CFD codes. To this end, both $2 \mathrm{D}$ and $3 \mathrm{D}$ ray-acoustic formulations are considered and compared. The governing equations are briefly discussed in $\$ 2$ with details relegated to the references. Section 3 describes two numerical examples. In the first example, the jet is modeled in closed form and the directivity angles and the sound pressure level are worked out using both 2D and 3D techniques. In order to extend the problem to supersonic jets, a second example is proposed in which the mean flow is calculated numerically. The jet under consideration is issued from a convergent-divergent nozzle at its design condition with a jet exit Mach number of $U_{j} / a_{\infty}=2.16$. We further require a shock-free flow at this point, as the propagation of rays across shocks discontinuities will require special treatment that is beyond the scope of the present work. The difficulties arising from the computation of the directivity factor of supersonic jets near the boundary of zone of silence are discussed. The Doppler factor in the direction of eddy Mach wave emission is compared with the conventional modified Doppler factor based on the line-ofsight method ${ }^{11,12}$. Finally the spherical directivity of an off-axis isotropic quadrupole convecting at various locations along the jet is calculated and compared with data. This is followed by summary and conclusions in the final section.

\section{Governing Equations}

It is well known that in a fine-grained turbulence, the retarded time across a turbulent eddy volume element is negligible. At subsonic convection Mach numbers, the radiation field arriving from each source volume element is independent of that due to any other region of the flow and the noise field is predicted by estimating the sound radiated by a typical correlation volume element. This is essentially done by Lighthill's equation. However, the interaction of the mean flow and its effect on the directivity pattern of the jet noise can be accounted for in the context of Lilley's equation; where the mean flow gradients as well as the source convection are moved to the operator part of the equation. In the high frequency approximation, as in geometrical optics, sound propagates along rays. Hence, the radiation pattern for each convecting multipole source is the result of the variation of the ray-tube area surrounding that ray. Therefore, rays emitted from each sound producing region of the flow need to be traced to an arbitrary observer point.

\subsection{Two-Dimensional Geometrical Acoustics}

For an inviscid parallel flow whose velocity $U$ and sound speed $a$ are arbitrary functions of the crossstream coordinates $X_{t}$, a pair of second order differential equations ${ }^{7}$ are solved to trace the rays of GA

$$
\begin{gathered}
\frac{d}{d s}\left(q \frac{d \mathbf{X}_{t}}{d s}\right)=\nabla_{t} q\left(\mathbf{X}_{t}\right) \\
q^{2}=\Phi^{2}\left(\mathbf{X}_{t}\right)-\cos ^{2} \theta_{\infty} \\
\Phi\left(\mathbf{X}_{t}\right)=\frac{1}{C\left(\mathbf{X}_{t}\right)}-M\left(\mathbf{X}_{t}\right) \cos \theta_{\infty} \\
M\left(\mathbf{X}_{t}\right)=\frac{U\left(\mathbf{X}_{t}\right)}{a\left(\mathbf{X}_{t}\right)}, \quad C\left(\mathbf{X}_{t}\right)=\frac{a\left(\mathbf{X}_{t}\right)}{a_{\infty}}
\end{gathered}
$$

subject to the initial conditions

$$
\left(\mathbf{X}_{t}\right)=\left(\mathbf{X}_{t}\right)_{o}, \quad \frac{d \mathbf{X}_{t}}{d s}=(\cos \delta, \sin \delta) \text { at } s=0 .
$$

In equations (1), $s$ is the arc length along the projection of the ray on a cross-stream plane (figure 1 ), $\delta$ is the radiation angle at the source, $(\theta, \phi)$ are polar and azimuthal angles in spherical coordinates and subscripts $o$ and $\infty$ refer to source and observer locations respectively. It is possible to solve (1) numerically as a system of four first order differential equations and continue along each ray until it becomes a straight line where $\phi_{\infty}$ is determined as a function of emission angle $\delta$. Thus for any pre-selected value of polar observation angle $\theta_{\infty}$, the spreading or focusing of acoustic rays is expressed as the azimuthal directivity factor $d \delta / d \phi_{\infty}$. The far field mean square pressure produced by point multipole sources convecting downstream at convection Mach $M_{c}=U_{c} / a_{\infty}$ is given $a^{7}$

$$
\begin{gathered}
\overline{P_{\mathcal{M}}^{2}}=\left\{\left(\frac{1}{4 \pi R}\right) \frac{\rho_{\infty} a_{\infty}}{a_{o}\left(1-\frac{U_{o}}{a_{\infty}} \cos \theta_{\infty}\right)\left(1-M_{r} \cos \theta_{\infty}\right)}\right\}^{2}\left(\frac{d \delta}{d \phi_{\infty}}\right) \\
\overline{P_{\mathcal{D}_{i}}^{2}}=\overline{P_{\mathcal{M}}^{2}}\left(\frac{k_{n}}{1-M_{e} \cos \theta_{\infty}}\right)^{2}\left|D_{i} \nu_{i}\right|^{2}
\end{gathered}
$$




$$
\begin{aligned}
& \overline{P_{\mathcal{Q}_{i j}}^{2}}=\overline{P_{\mathcal{M}}^{2}}\left(\frac{k_{\circ}}{1-M_{\circ} \cos \theta_{\infty}}\right)^{4}\left|Q_{i j} \nu_{i} \nu_{j}\right|^{2} \\
& \nu_{1}=q_{\circ} \cos \delta, \quad \nu_{2}=q_{\circ} \sin \delta, \quad \nu_{3}=\cos \theta_{\infty}
\end{aligned}
$$

where $\overline{P_{\mathcal{M}}^{2}}$ is the radiation field due to a monopole source of unit strength, $\overline{P_{\mathcal{D}_{i}}^{2}}$ and $\overline{P_{\mathcal{Q}_{i j}}^{2}}$ denote the fields due to dipole and quadrupole sources of various orientations and $D_{i}\left(\omega_{0}\right)$ and $Q_{i j}\left(\omega_{0}\right)$ are the dipole and quadrupole source strength respectively. In (2) $k_{n}$ is the wave number $\left(k_{o}=\omega_{0} / a_{\infty}\right)$ and the source frequency $\omega_{0}$ is related to the observer frequency $\omega$ through the familiar Doppler factor

$$
\omega=\frac{\omega_{0}}{1-M_{e} \cos \theta_{\infty}} .
$$

When the quadrupole source is isotropic $\left(Q_{i j}=Q_{n}\right.$ for $i, j=1,2,3)$, it can be concluded that

$$
\overline{P_{Q_{0}}^{2}} \propto\left(\frac{\rho_{\infty} a_{\infty}}{4 \pi R}\right)^{2} \frac{Q_{0}^{2} \omega_{0}^{4}}{a_{0}^{6}} \frac{\left(1-\frac{U_{0}}{a_{\infty}} \cos \theta_{\infty}\right)^{2}}{\left(1-M_{r} \cos \theta_{\infty}\right)^{5}} \frac{d \delta}{d \phi_{\infty}} .
$$

A Doppler factor correction for source volume effects ${ }^{11}$ has been utilized in (4).

\subsection{Three-Dimensional Geometrical Acoustics}

For an inviscid flow governed by linearized gas dynamic equation, with mean velocity $\mathbf{U}(\mathbf{X})$ and sound speed $a(\mathbf{X})$, the propagation of high frequency noise is governed by

$$
\begin{gathered}
\frac{d X_{i}}{d s}=T_{i j} p_{j}+M_{i} / C \quad i, j=1,2,3 \\
\frac{d p_{i}}{d s}=-\frac{1}{2} p_{j} \frac{\partial T_{j k}}{\partial X_{i}} p_{k}-p_{j} \frac{\partial}{\partial X_{i}}\left(\frac{M_{j}}{C}\right)+\frac{1}{2} \frac{\partial}{\partial X_{i}}\left(C^{-2}\right) \\
T_{i j}=\delta_{i j}-M_{i} M_{j}
\end{gathered}
$$

where $M_{i}$ denotes the Mach component $U_{i} / a, p_{i}$ is the component of phase normal $\mathbf{p}$ and $C(\mathbf{X})$ is the normalized sound speed defined earlier. Equations (5a) are numerically integrated along the ray subject to the initial conditions

$$
\begin{gathered}
\mathbf{X}=\mathbf{X}_{0} \quad \text { at } \quad s=0 \\
\mathbf{p}_{o}=\lambda \hat{\mathbf{X}}+\left(\lambda \hat{\mathbf{X}} \cdot \mathbf{M}-\frac{1}{C}\right) \frac{\mathbf{M}}{\beta^{2}} .
\end{gathered}
$$

The ray speed $\lambda=|d \mathbf{X}| / d s$ is given as

$$
\lambda=\left[\beta^{2}+(\hat{\mathbf{X}} \cdot \mathbf{M})^{2}\right]^{-\frac{1}{2}} / C
$$

$$
\beta^{2}=1-|\mathbf{M}|^{2}
$$

where unit vector $\hat{\mathbf{X}}_{0}=(\cos \mu, \sin \mu \cos \delta, \sin \mu \sin \delta) \mathrm{de}-$ notes the direction of emission at the source (figure 2).

Angle $\mu$ is measured with respect to a stationary frame of reference and is simply the vector sum of the direction of emission in the source coordinate and the mean velocity vector. For subsonic jets $\left(\left|M_{0}\right|<1\right)$ this angle could clearly become as large as $\pi$, however, for a supersonic flow $\left(\left|M_{n}\right|>1\right)$ the upper limit on angle $\mu$ is less than $\pi / 2$, resulting in a real value for the ray speed $\lambda$ in (5c). When the ray becomes straight $\lambda$ approaches one and a pair of angles $\left(\theta_{\infty}, \phi_{\infty}\right)$ are calculated for every pair of pre-selected $(\mu, \delta)$. The far field mean square pressure directivity for a convecting multipole source of frequency $\omega_{n}$, accounting for source volume effects becomes ${ }^{9,10}$

$$
\begin{gathered}
\overline{P_{\mathcal{M}}^{2}} \propto\left(\frac{\rho_{\infty} a_{\infty}}{4 \pi R}\right)^{2}\left(\frac{\lambda_{0}^{3}}{\rho_{o}} \frac{\sin \mu}{\sin \theta_{\infty}} \frac{a_{o}^{4}}{\left|\frac{\partial\left(\theta_{\infty}, \phi_{\infty}\right)}{\partial(\mu, \delta)}\right|}\right) \times \\
\frac{\left(1-\frac{\mathrm{U}_{\infty}}{a_{\infty}} \cdot \mathbf{p}_{\infty}\right)^{2}}{\left(1-\frac{\mathrm{U}_{\infty}}{a_{\infty}} \cdot \mathbf{p}_{n}\right)^{2}\left(1-\frac{\mathrm{U}_{c}}{a_{\infty}} \cdot p_{o}\right)} \\
\overline{P_{\mathcal{D}_{i}}^{2}}=\overline{P_{\mathcal{M}}^{2}}\left(\frac{k_{o}}{1-\frac{\mathrm{U}_{c}}{a_{\infty}} \cdot \mathbf{p}_{n}}\right)^{2}\left|\left(p_{o}\right)_{i} D_{i}\right|^{2} \\
\overline{P_{\mathcal{Q}_{i j}}^{2}}=\overline{P_{\mathcal{M}}^{2}}\left(\frac{k_{o}}{1-\frac{\mathrm{U}_{c}}{a_{\infty}} \cdot \mathbf{p}_{o}}\right)^{4}\left|\left(p_{o}\right)_{i}\left(p_{o}\right)_{j} Q_{i j}\right|^{2}
\end{gathered}
$$

Here the source and observer frequencies are related as

$$
\omega=\omega_{n} /\left(1-\frac{U_{c}}{a_{\infty}} \cdot p_{n}\right)
$$

For an isotropic quadrupole source $Q_{i j} p_{i} p_{j}=Q_{0}|\mathbf{p}|^{2}$ and the sound field for a source of strength $Q_{n}\left(\omega_{0}\right)$ becomes

$$
\begin{gathered}
\overline{P_{\mathcal{Q}_{0}}^{2}} \propto\left(\frac{\rho_{\infty} a_{\infty}}{4 \pi R}\right)^{2} Q_{n}^{2} \omega_{n}^{4}\left(\frac{a_{o}^{2}}{a_{\infty}^{2}} \frac{\lambda_{o}^{3}}{\rho_{n}}\right) \times \\
\frac{\left(1-\frac{U_{0}}{a_{\infty}} \cdot \mathbf{p}_{n}\right)^{2}\left(1-\frac{U_{\infty}}{a_{\infty}} \cdot \mathbf{p}_{\infty}\right)^{2}}{\left(1-\frac{U_{0}}{a_{\infty}} \cdot \mathbf{p}_{n}\right)^{5}}\left(\frac{\sin \mu}{\sin \theta_{\infty}} \frac{1}{\left|\frac{\partial\left(\theta_{\infty}, \phi_{\infty}\right)}{\partial(1, \delta)}\right|}\right) .
\end{gathered}
$$

In the far field $U_{\infty}$ is usually small. It is shown in the Appendix that $\lambda_{0}^{3} / \rho_{n}$ is of the order of $a_{0}^{-8}$. It appears that equations (4) and (8) are essentially similar except for the directivity factor $d \delta / d \phi_{\infty}$ which is replaced with its 3D counterpart in equation (8). In addition, the 3D Doppler factor is influenced by the refraction phenomenon, as there is a unique phase normal $p_{0}$ at the source which results in propagation of a ray through 
an observer point. It will be shown later that the singularity in (3) in the direction of eddy Mach emission $\left(\theta_{\infty}=\cos ^{-1} 1 / M_{c}\right)$ does not appear in (7). In fact we will compare the two expressions and assess the noncompactness constant of the modified Doppler factor.

\section{Numerical Results}

In order to integrate the propagation equations (1) and (5) it is necessary to specify the spatial dependencies of the mean jet velocity and temperature. A closed form formulation will naturally speed up the computation of the gradients. However, when the jet is calculated numerically from the commonly available CFD codes, it is possible to integrate the CFD solution with an appro priate interpolation routine (2D interpolation for axisymmetric jets and 3D for non-axisymmetric) and fit a mutidimensional function on data at the grid points. Having found the tensor coefficients of the interpolating function, the directional derivatives at any point within the flow is readily available.

\subsection{Closed form Jet Model}

An axisymmetric self-similar Mach profile can be written in spherical coordinates as

$$
\frac{\mathrm{U}(R, \theta)}{a(R, \theta)}=\left(\frac{U}{a}\right)_{C L} e^{-\left(\frac{\ell}{\alpha}\right)^{n}}
$$

where $U$ is in the direction of $\theta, \alpha$ is the jet spreading angle and $(U / a)_{C L}$ denotes the centerline value of the Mach number. For convenience, the velocity decay on the centerline is expressed as a function of the axial distance from the exit plane ${ }^{10}$. Further, in the case of a cold jet (constant stagnation temperature), the sound speed is expressed using adiabatic flow relationship. For a subsonic jet with exit Mach number of $U_{j} / a_{\infty}=0.99$, the radiated noise for an off-axis source was worked out in Ref. 10. In that exercise, the jet spreading angle was $\alpha=10^{\circ}$ and a 3D ray-acoustic approach was employed to assess the directivity of a convecting ring source at 7 diameters from the exit.

For the purpose of comparison with the $2 \mathrm{D}$ predictions, we now let the jet spreading angle be very small $\left(\alpha=1^{\circ}\right)$, while the streamwise evolvement of the flow expressed through $n\left(X_{1}\right)$ is kept intact ( see Ref. 10). Thus, in the context of 2D-GA, the flow is more or less a quasi$3 D$ jet. This is due to fact that rays obtained according to (1) are representative of the flow characteristics on a spanwise plane at the source location. By moving the source to a new axial location the surrounding flow is allowed to change accordingly.

\subsubsection{Directivity Angles}

Figure 3 shows emission angle $\delta$ v.s. azimuthal angle $\phi_{\infty}$ with polar angle $\theta_{\infty}$ indicated as a parameter for an off-axis source at $x_{n} / D=7$ and $r_{n} / D=0.31$. A proper selection of the target angle $\theta_{\infty}$ is essential in solving (1). There is a minimum allowable angle for any source point found by setting $q_{n}^{2}=0$, which gives $\theta^{\star}=\cos ^{-1} 1 / C_{0}\left(1+M_{0}\right)$ as the boundary of the zone of silence. An attempt to solve the propagation equations with $\theta_{\infty}<\theta^{\star}$ will trap the rays within the envelope of $q_{n}^{2}=0$ as depicted in figure 4 . The boundary of the zone of silence for this source is at $53.7^{\circ}$ and figure 4 was obtained for $\theta_{\infty}=50.0^{\circ}$. When $\theta_{\infty}>\theta^{\star}$ but still close to $\theta^{\star}$, the rays are confined to a narrow azimuthal angle $\phi_{\infty}$ as seen in figure 3 for $\theta_{\infty}<55^{\circ}$. This is due to a complete refraction of rays as will be shown clearly in 3D-GA. In addition, it appears that within this range of $\theta_{\infty}$ two rays could be arriving from a single source. However, the 3D directivity angles indicate that the two rays indeed arrive at slightly different polar angles. Our 3D ray-acoustic computations for the same source point are shown in figure 5 , where observer angles are given as a function of radiation angles at the source. Most of the radiated energy is limited to a narrow range of $\theta_{\infty}$, near the zone of silence (figure $5 \mathrm{~b}$ ). An enhanced version of figure $5 \mathrm{~b}$ is given in $5 \mathrm{c}$, showing slight variation in $\theta_{\infty}$ with $\delta$ resulting in a single ray arrival from each source point. Here the boundary of zone of silence was $52.7^{\circ}$, differing from the $2 \mathrm{D}$ predictions by only one degree due to non-parallel effects. In the limit, as the flow becomes parallel, $\theta_{\infty}$ becomes a function of $\mu$ only

$$
\cos \theta_{\infty}=\frac{\frac{\cos ^{\prime} \mu}{\sqrt{1-M_{o}^{2} \text { in }^{2} \mu}}-M_{0}}{C_{0}\left(1-M_{0}^{2}\right)}
$$

and with $\mu=0$ the boundary of zone of silence reduces to that of $2 \mathrm{D}$ ray-acoustic described earlier. For the purpose of completeness, we point out that figure 3 is, more or less, $a$ direct conclusion of figures $5 a$ and $5 b$. It is seen from figure $5 \mathrm{~b}$ that if the variation of $\theta_{\infty}$ with $\delta$ is neglected, parameter $\mu$ will be associated with a single $\theta_{\infty}$ (say $\theta_{\infty}$ at $\delta=0$ ). Eliminating cone angle $\mu$ will combine $5 \mathrm{a}$ and $5 \mathrm{~b}$ into figure 6 which shows a remarkable resemblance to the directivity angles of figure 3 .

\subsubsection{Sound Pressure Level Directivity}

We continue our comparison of the $2 D$ and $3 D$ rayacoustic methods with the computation of the noise directivity for a convecting isotropic quadrupole source as given in equations (4) and (8). Although a prior knowledge of the source strength and its frequency is a requirement, for our purpose we limit the discussion to a point source or a ring source within an axisymmetric flow and leave out dependence on $Q_{n}^{2} \omega_{n}^{4}$. The convection velocity $U_{e}$ in the most energetic part of the shear 
layer is close to the jet mean velocity (Ref. 13) which leads one to believe that the high frequency noise scales as -3 power of the Doppler factor. In all the following computations, however, the convection Mach is expressed as a weighted average of the local and exit Mach numbers (referenced with respect to ambient sound speed) as $\mathrm{U}_{c} / a_{\infty}=0.5 \mathrm{U}_{o} / a_{\infty}+0.3 \mathrm{U}_{j} / a_{\infty}$. Figures $7 \mathrm{a}, 7 \mathrm{~b}$ and $7 \mathrm{c}$ show the sound pressure level directivity for an offaxis quadrupole point source at 7 diameters from the jet exit. On approaching the boundary of the zone of silence, the $2 \mathrm{D}$ directivity factor becomes infinitely large (figure 7a). A 3D ray-acoustic prediction (figure 7c), on the other hand, gives a finite value for the directivity factor as both $\sin \mu$ and $\left|\frac{\partial\left(\theta_{\infty}, \phi_{\infty}\right)}{\partial(\mu, \delta)}\right|$ become small. Further away from the zone of silence $\left(\theta_{\infty} \geq 57^{\circ}\right)$, there is close agreement between the two predictions. A different view of the spherical directivity for this source point is shown in figures $8 \mathrm{a}$ and $8 \mathrm{~b}$.

The sound emission from a ring source is the direct result of integration of figure 7 for $0<\phi_{\infty}<2 \pi$ and is shown in figure 9 . Here, the 2D calculation were terminated close to the zone of silence where the $3 \mathrm{D}$ predictions result in a sudden drop within the zone of silence. While the decay rate within the sound valley will decrease with increasing the jet spreading angle, still the high frequency approximation predicts a faster decay compared with measurements. Ref. 10 compares data with the ring source predictions for this jet at a spreading angle of $\alpha=10^{\circ}$.

\subsection{Supersonic Jets}

The geometry considered for this exercise is an axisymmetric convergent-divergent nozzle. The flowpath is designed to generate a uniform and parallel flow at the nozzle exit for the design Mach number of 1.4 (figure 10a). Both aerodynamic and acoustic data ${ }^{14}$ were available for comparison with predictions. For this hot jet the stagnation temperature was $1716^{\circ} R$ which results in an exit Mach number of $M_{j}=U_{j} / a_{\infty}=2.16$. An axisymmetric version of the PARC code with Chien's $k-\epsilon$ turbulence mode ${ }^{16}$ was employed to solve the complete Reynolds-averaged Navier Stokes equations in conservative law form. The predicted flow field ${ }^{17}$ was shown to be in good agreement with data of Ref. 14. A third order spline fit was used to interpolate a surface on the CFDgenerated flow field at the grid points $(91 \times 60$ points). The spline fit for the normalized sound speed $C(\mathbf{X})$ is shown in figure 10b. For each one of the four variables of interest (sound speed and the three components of Mach vector) the tensor coefficients of the interpolating functions were passed to a subroutine that computes the directional derivatives.

\subsubsection{Directivity Angles}

Figure 11 shows the 3D predictions for the boundary of the zone of silence for this supersonic jet as a function of source location. In a fine-grained turbulence, the acoustically active sources are associated with the kinetic energy of turbulence which is primarily distributed around the lip-line of the jet $\left(r_{0} / D=0.5\right)$. We will see shortly that for supersonic jets the radiation from any source point is, more or less, concentrated in a narrow range of polar angle near the zone of silence of that source. It is therefore reasonable to conclude that on the basis of figure 11 peak noise for this jet is in direction of $42^{\circ}<\theta_{\infty}<50^{\circ}$, in agreement with experimental data ${ }^{14}$.

The radiation by turbulence convected at supersonic speed was termed by Phillips $^{18}$ as eddy Mach wave emission. The Mach waves are believed to be emitted from the shear layer at an angle of $\theta=\cos ^{-1}\left(\frac{a_{0}}{\beta U_{j}}\right)$ where $\beta$ is a constant generally taken as 0.7 . This results in a Mach angle of $\theta=48.5^{\circ}$ for the present jet. Another important feature of supersonic jets is related to source efficiency. As has been pointed out by Ffowcs Williams ${ }^{19}$ the individual quadrupole sources essentially radiate as monopoles with no near field cancellation. This increased efficiency is capable of converting between 0.1 to $1 \%$ of the mechanical energy to noise as opposed to $.01 \%$ at angles beyond this range.

The $3 \mathrm{D}$ directivity angle for an off-axis source at $x_{n} / D=7$ and $r_{n} / D=0.5$ is shown in figure 12. An enhanced version of figure $12 \mathrm{~b}$ is shown in $12 \mathrm{c}$ where the slight variation of $\theta_{\infty}$ with $\delta$ is noticeable. A distinct zone of silence at $\theta^{\star}=48.3^{\circ}$ is seen clearly. With equal increments of $5^{\circ}$ in the emission angle $\mu$, the radiated energy is shown to be concentrated at a narrow angle near $\theta^{\star}$. As $\mu \rightarrow 0$, there is a possibility for both $\frac{\partial \theta_{\infty}}{\partial \delta}$ and $\frac{\partial \phi_{\infty}}{\partial \delta}$ to become increasingly small. When this occurs the directivity factor will approach $\frac{0}{0}$ but it is possible to resort to higher order derivatives at the cost of an increase in the required precision in the directivity angles.

To assess the performance of the 2D-GA, the directivity angles for the same source point within the supersonic jet is shown in figure $12 \mathrm{~d}$. The zone of silence is given as $66.7^{\circ}$, not anywhere close to the previous prediction. Presumably the non-parallel effects play a major role in refraction of acoustic energy at supersonic speeds.

\subsubsection{Noise of Supersonic Jets}

In the last section, we briefly addressed the role of fine-grained turbulence in the noise of supersonic jets. It was shown that noise is primarily radiated in a narrow angle near the zone of silence. When the jet is not perfectly expanded, shock-associated noise and screech tones 
are generated due to interaction of turbulence with the shock cells. Here we concentrate on the mixing noise as described through equations (6) and (8). An examination of equation (6) indicates that the monopole field $\overline{P_{\mathcal{M}}^{2}}$ is of the order -3 power of the Doppler factor, as is equation (8). Leaving out the source strength, both equations produce similar directivity patterns.

Figures 13a \& 13b show the spherical directivity of the radiated noise for an off-axis source, with parameter $\phi_{\infty}$ indicating the relative azimuthal angle between the source and the observer. As was pointed out earlier, a high clustering of rays occurs within a narrow polar angle at the boundary of the zone of silence, resulting in increased difficulty in the computation of the directivity factor.

The role of refraction and its interaction with the Doppler factor for supersonic jets is demonstrated by comparing the denominators of equations (3) and (7) in figure 14a. Due to the singular behavior of the Doppler factor, Ffowcs Williams ${ }^{11}$ and Ribner ${ }^{12}$ suggested a modified Doppler factor $\mathcal{C}^{m}=\left\{\left(1-M_{r} \cos \theta_{\infty}\right)^{2}+\alpha^{2} M_{r}^{2}\right\}^{0.5}$. The noncompactness constant $\alpha$ is a semiempirical constant of the order of $0.3-0.55$. Here we compute $\alpha$ directly from

$$
\mathcal{C}^{m}=\left(1-\frac{\mathrm{U}_{c}}{a_{\infty}} \cdot \mathbf{p}_{o}\right)
$$

The results are shown in figures $14 \mathrm{~b} \& 14 \mathrm{c}$. It is seen from $14 \mathrm{~b}$ that $\alpha$ is angle dependent, approaching 0.4 for the most energetic part of the radiation field. A more accurate representation of $\alpha$ is given in figure $14 \mathrm{c}$ as a function of the radiation angle $\mu$. The reader may recall that in our model the eddy convection Mach is a function of the source location. By moving closer to the axis $\left(r_{n} / D=\right.$ 0.25 ), the Doppler factors compare as seen in figure 15a resulting in $\alpha=0.33$ shown in figures $15 \mathrm{~b} \& 15 \mathrm{c}$. Overall, by investigating various source locations within the jet, it appears that the suggested value for $\alpha$ is indeed a good approximation.

We show the directivity of the ring source in figure 16a. The scaling of the directivity with power -3 of the Doppler factor is also shown in this figure as

$$
\mathcal{D}\left(\theta_{\infty}\right)=\int_{0}^{2 \pi} \frac{\left(1-\frac{U_{c}}{P_{\infty}^{2}} \cdot p_{n}\right)^{5}}{\left(1-\frac{U_{\infty}}{a_{\infty}} \cdot p_{n}\right)^{2}} d \phi_{\infty}
$$

where the integrand is calculated at a point on the ring. Moving the ring source closer to the jet axis will result in directivity figure $16 \mathrm{~b}$.

Our ring source predictions are also compared with data of Ref. 14 as seen in figure 17. It appears that the most important features of the noise directivity of this supersonic jet are captured through the ring source elements selected in this exercise. An integration of equation (8) for the entire jet can be worked out with proper knowledge of the source intensity spectra.

\section{Conclusions}

In this paper, applications of geometrical acoustics to propagation of high frequency jet noise was discussed. The propagation equations were solved using both 2D \& 3D ray-acoustic models. It was demonstrated that at subsonic Mach numbers, the $2 \mathrm{D}$ analysis results in satisfactory predictions outside the zone of silence, and therefore should be considered a viable substitute for the more complicated 3D computations. Unlike the subsonic jets, the parallel flow assumption proves to be inadequate for high speed jets.

By computing the mean flow using a CFD code, it was shown that the directivity factor can indeed be calculated for jets of arbitrary geometries. Assuming that the sources of noise are embedded within the mean flow, we showed that refraction phenomenon modifies the supersonic Doppler factor significantly and results in a nonsingular pattern in the direction of eddy Mach wave emission. These arguments were used to assess the modified Doppler factor based on the line-of-sight method.

The extension of the problem to non-axisymmetric jets seems relatively straightforward, although the increase in computational requirements suggests that perhaps a number of jet slices should be included in the volume integration. In addition, such an attempt will also require the magnitude of the source strength of the nonaxisymmetric jet.

\section{References}

${ }^{1}$ Lilley, M. G., "On the Noise from Jets," AGARD CP131, 1973.

${ }^{2}$ Mani, R., "The Influence of Jet Flow on Jet Noise, Part I, The Noise of Unheated Jets," J. Fluid Mech., Vol. 76, 1976, pp. 753-778.

${ }^{3}$ Mani, R. "The Influence of Jet Flow on the Jet Noise, Part II, The Noise of Heated Jets," J. Fluid Mech., Vol 76, 1976, pp. 779-793.

${ }^{4}$ Balsa, T. F., "The Far Field of High Frequency Convected Singularities in Sheared Flows, with an Application to Jet Noise Prediction," J. Fluid Mech., Vol. 74, 1976, pp. 193-208.

${ }^{5}$ Tester, B. J. and Morfey, C. L., "Developments in Jet Noise Modeling - Theoretical Predictions and Comparisons with Measured Data," J. Sound Vib., Vol. 46(1), 1976, pp. 79-103.

${ }^{6}$ Scott, J. N., "Propagation of Sound Waves Through Linear Shear Layer," AIAA Journal, Vol. 17, 1979, pp. 237-244. 
${ }^{7}$ Goldstein, M. E., "High Frequency Sound Emission from Moving Point Multipole Sources Embedded in Arbitrary Transversely Sheared Mean Flows," J. Sound Vib., Vol. 80(4), 1982, pp. 499-522.

${ }^{8}$ Durbin, P. A., "High Frequency Green Function for Aerodynamic Noise in Moving Media, part I: General Theory," J. Sound Vib., Vol. 91(4), 1983, pp. 519-525.

${ }^{9}$ Durbin, P. A., "High Frequency Green Function for Aerodynamic Noise in Moving Media, part II: Noise from a Spreading Jet," J. Sound Vib., Vol. 91(4), 1983, pp. $527-538$.

${ }^{10}$ Khavaran, A. and Krejsa, E. A., "Propagation of High Frequency Jet Noise Using Geometric Acoustics," AIAA Paper 93-0147, 1993.

${ }^{11}$ Ffwocs Williams, J. E., "The Noise from Turbulence Convected at High Speeds," Aeronautical Research Council (Britain), ARC 23, 323-N, 184-FM 3138, 1961; superseded by Phil. Trans. Roy. Soc. of London, Vol. A255, 1963, pp. $469-503$.

${ }^{12}$ Ribner, H. S., "Aerodynamic Sound from Fluid Dilatations: A Theory of Sound from Jets and Other Flows," Univ. of Toronto, Institute for Aerospace Studies, Rept. 86, AFOSR TN 3430, 1962.

${ }^{13}$ Davies, P. O. A. L., Fisher, M. J., and Barratt, M. J., "The Characteristics of the Turbulence in the Mixing Region of a Round Jet," J. Fluid Mech., Vol. 15, 1963, pp. 337-367.

${ }^{14}$ Yamamoto, K. J., et al, "Experimental Investigation of Shock-Cell Noise Reduction for Single Stream Nozzles in Simulated Flight - Comprehensive Data Report," NASA CR-168234, 1984.

${ }^{15}$ Cooper, G. K. and Sirbaugh, J. R., "PARC Code: Theory and Usage", AEDC-TR-89-15, 1989.

${ }^{16}$ Chien, K. Y., "Prediction of Channel and Boundary Layer Flows with a Low Reynolds-Number Turbulence Model," AIAA Journal, Vol. 20, Jan. 1982, pp. 33-38.

${ }^{17}$ Khavaran, A., Krejsa, E. A. \& Kim, C. M., "Computation of Supersonic Jet Mixing Noise for an Axisymmetric CD Nozzle Using $k-\epsilon$ Turbulence Model," AIAA Paper 92-0500, 1992.

${ }^{18}$ Phillips, O. M., "On the Generation of Sound by Supersonic Turbulent Shear Layers," J. Fluid Mech., Vol. 9, 1960 , pp. 1-28,

${ }^{19}$ Ffwocs Williams, J. E. and Maidanik, G., "The Mach Wave Field Radiated by Supersonic Turbulent Shear Flows," J. Fluid Mech., Vol. 2, 1965, pp. 641-657.

\section{Appendix}

We approximate $\lambda_{0}$ assuming that the mean flow is in $X_{1}$ direction, i.e., $\mathbf{M}=M \hat{\mathbf{i}}$, and that the relative azimuthal separation between the direction of emission and the observer is zero, i.e., $\hat{\mathbf{X}}_{o}=(\cos \mu, \sin \mu, 0)$. From equation (5c) we have

$$
\lambda_{n}^{-1}=\frac{a_{n}}{a_{\infty}} \sqrt{1-\left(M_{n} \sin \mu\right)^{2}} .
$$

The term $M_{0} \sin \mu$ is usually smaller than one. Even when the jet becomes supersonic, i.e. $M_{0}>1$, most of the radiated energy is limited to small values of the emission angle $\mu$; hence $\lambda_{0}^{-1} \simeq \frac{a_{0}}{a_{\infty}}$. Using the one-dimensional gas dynamic relation for an adiabatic gas we have

$$
\frac{\rho_{0}}{\rho_{\infty}} \simeq\left(\frac{a_{o}}{a_{\infty}}\right)^{\frac{2}{\gamma-1}} \text {. }
$$

With $\gamma=1.4$ it is seen that $\left(\frac{\rho_{\infty}}{\rho_{0}}\right) \lambda_{a}^{3} \simeq\left(\frac{a_{\infty}}{a_{0}}\right)^{8}$. 


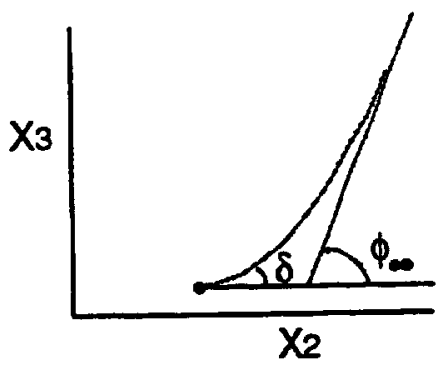

Figure 1.-Projection of a ray on cross-stream plane.
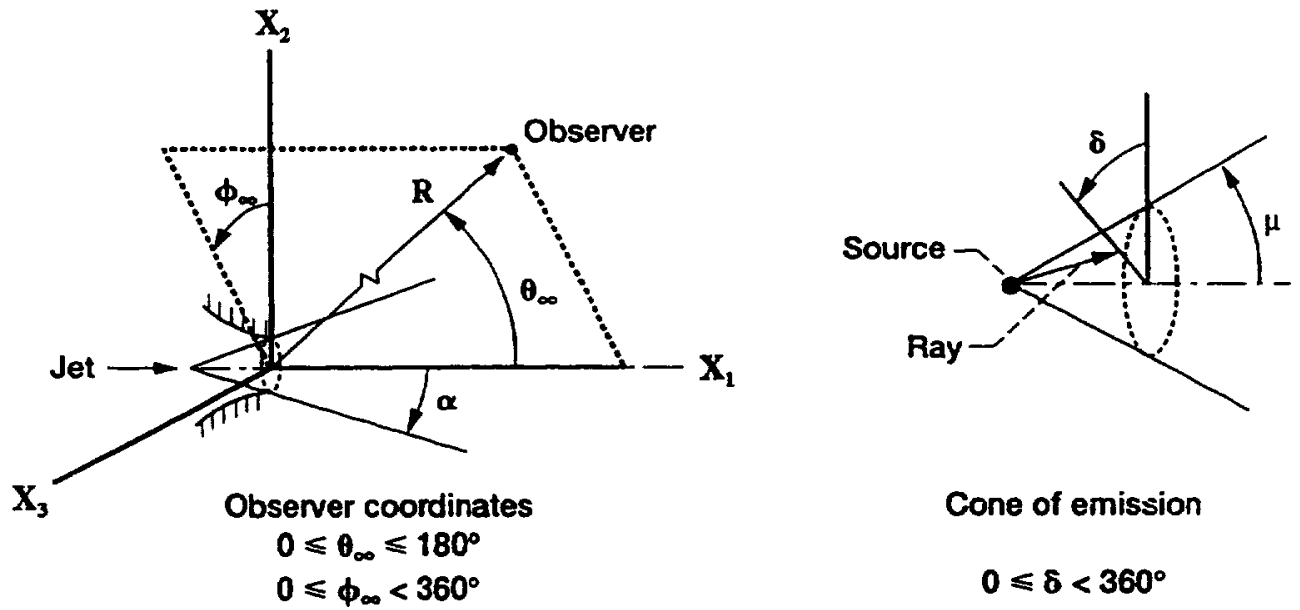

Cone of emission

$0 \leqslant \delta<360^{\circ}$

Figure 2.-Source and observer coordinates.

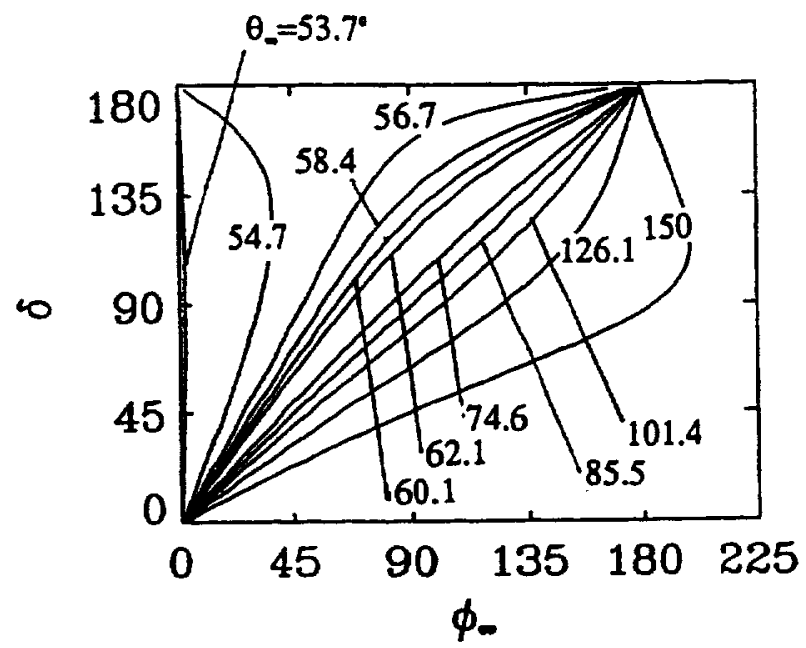

Figure 3.-Directivity angles for an off-axis source obtained from $2 D-G A$ with $M_{J}=0.99, X_{0} D=7$ and $r_{0} D=0.31$. 


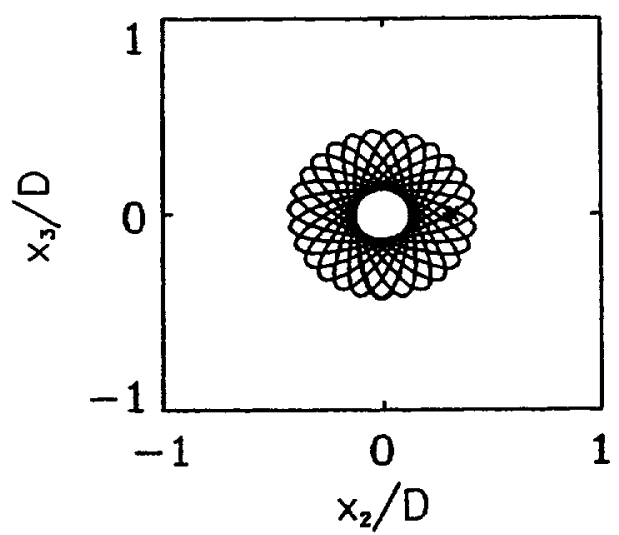

Figure 4.-Cross-stream projection of a ray when the target angle $0_{\infty}$ is within the zone of silence of the source; $X_{0} / D=7, r_{0} / D=0.31$, $\theta_{\infty}=50^{\circ}$ and $\delta=31^{\circ}$.
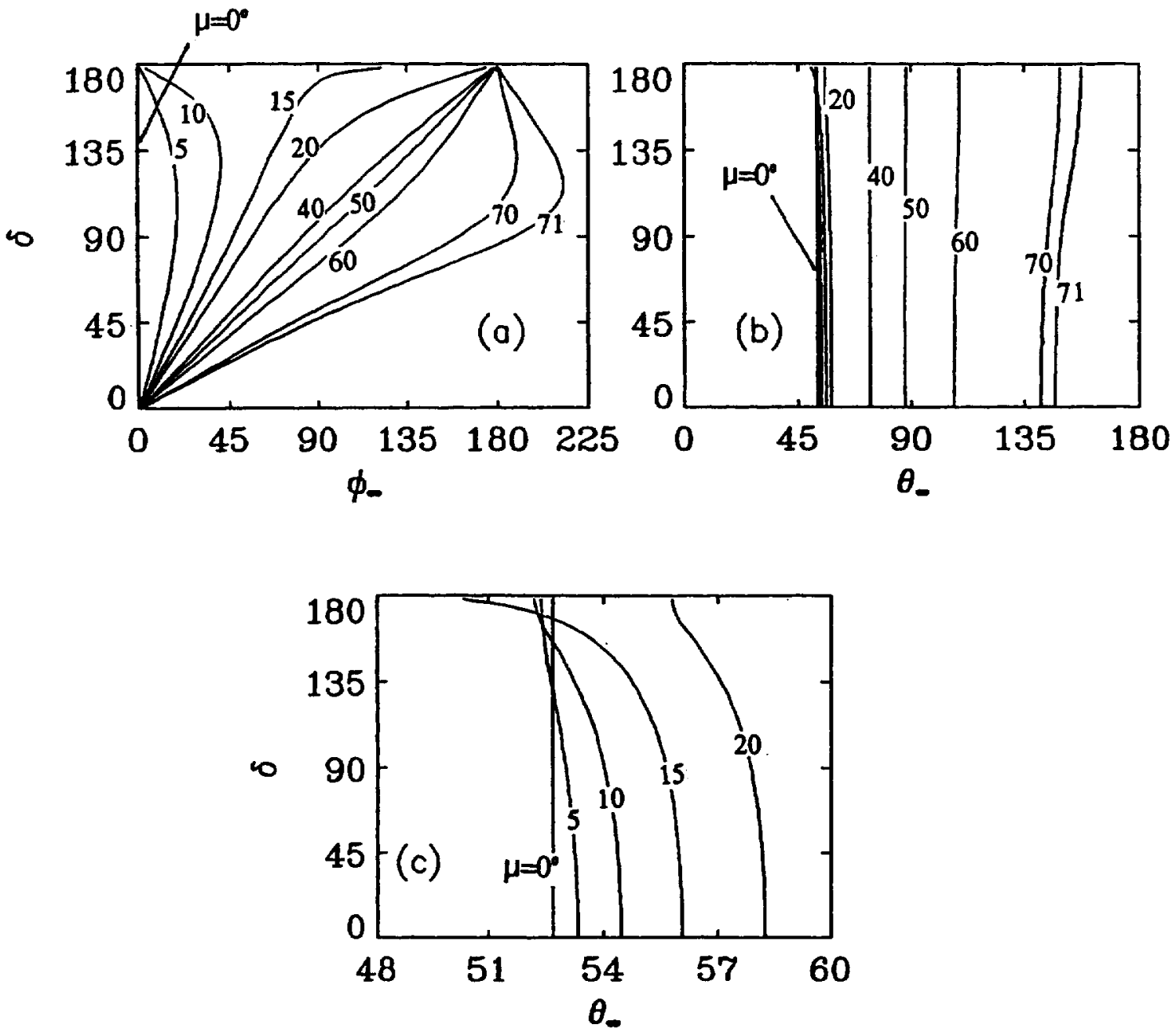

Figure 5.-Directivity angles $\left(\phi_{\infty}, \theta_{\infty}\right)$ vs emission angles $(\delta, \mu)$. Figure $(c)$ is enlarged $(b)$. The source is at $X_{0} / D=7$, $r_{0} / D=0.31$ with $M_{J}=0.99$. 


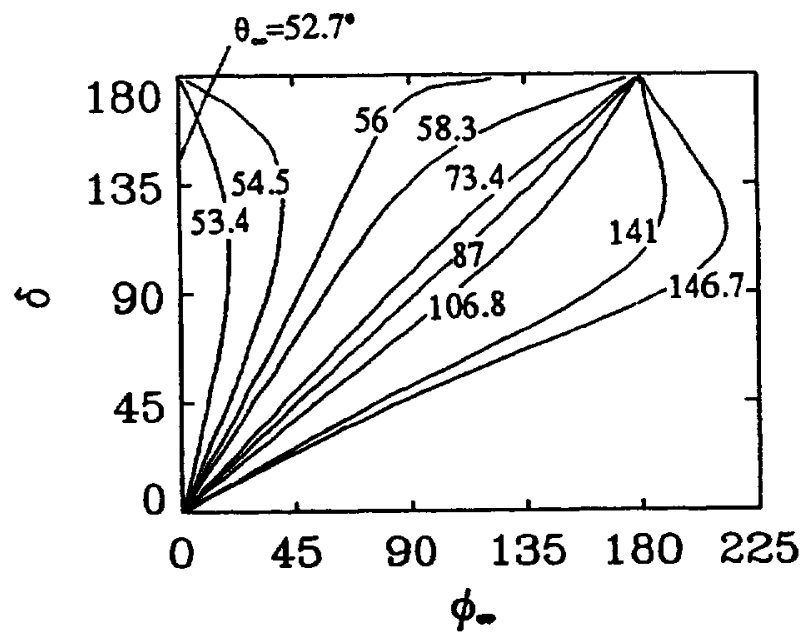

Figure 6.- Combination of figures $(5 b)$ and $(5 c)$ when variation of $0_{\infty}$ with 8 is neglected. 


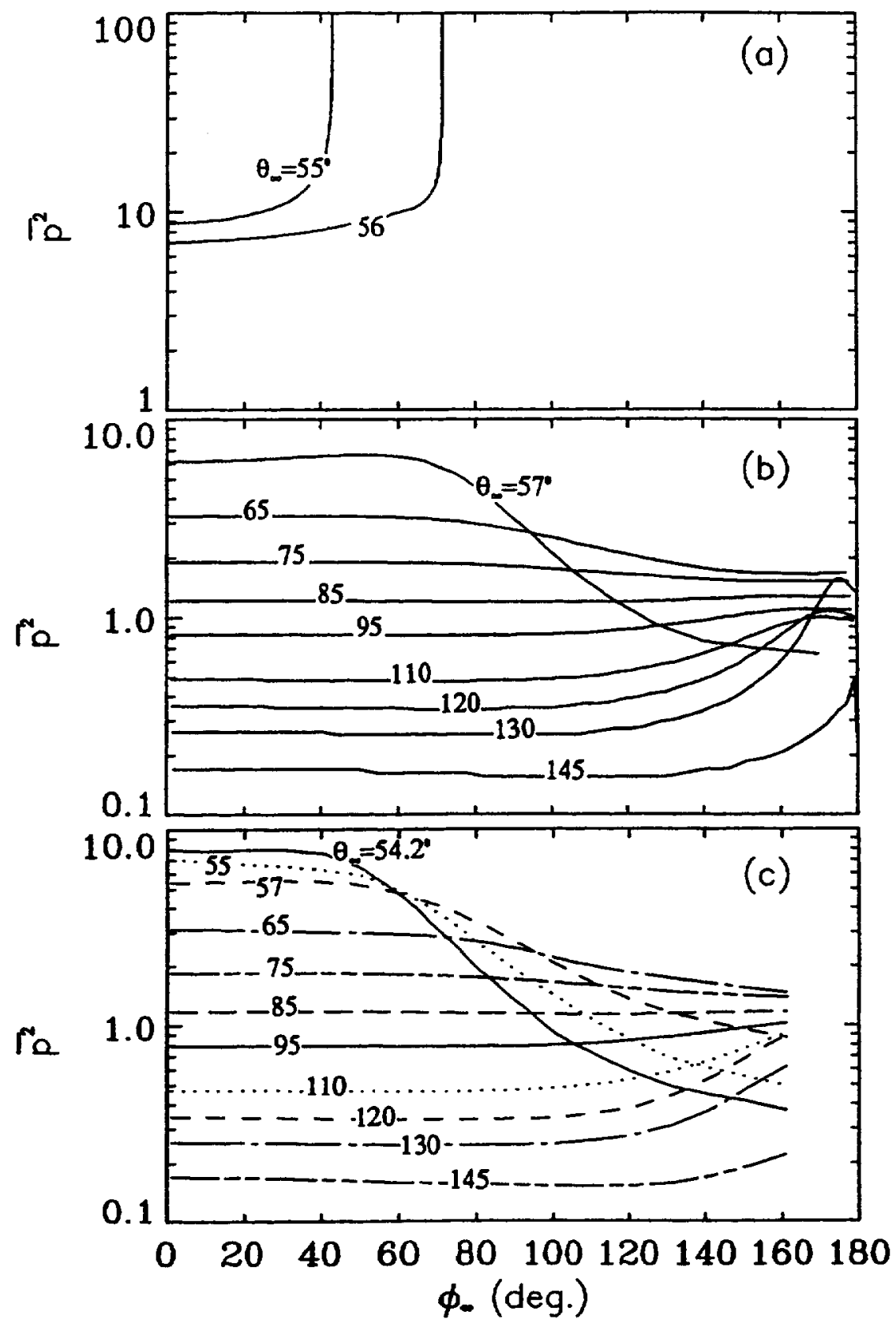

Figure 7. - Sound pressure level directivity for an off-axis source. Figures (a) and (b) are the 2D-GA predictions. Figure (c) is the 3D-GA prediction. 


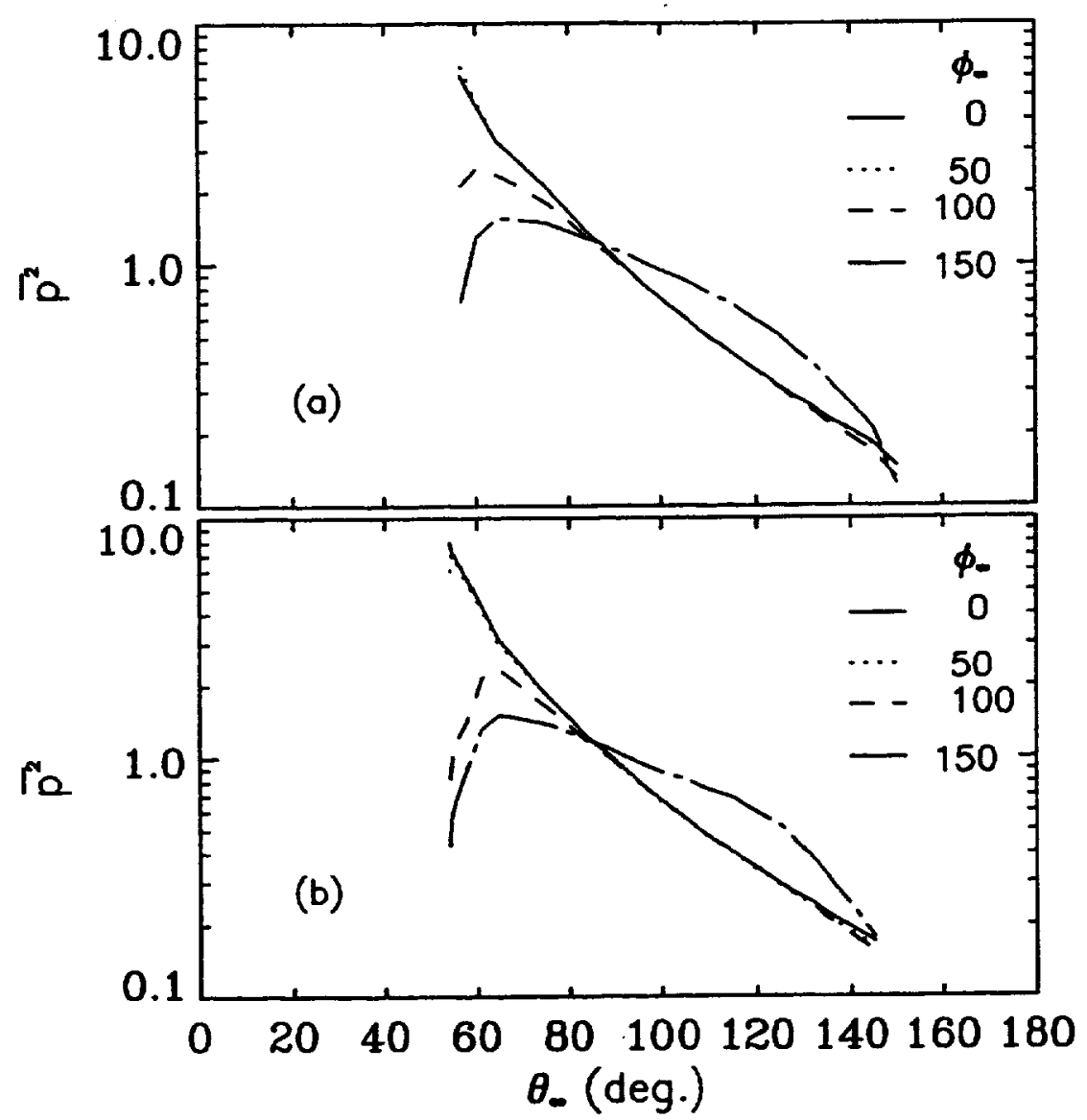

Figure 8. Polar directivity for the source of figure 7. (8a) 2D-GA. (8b) 3D-GA.

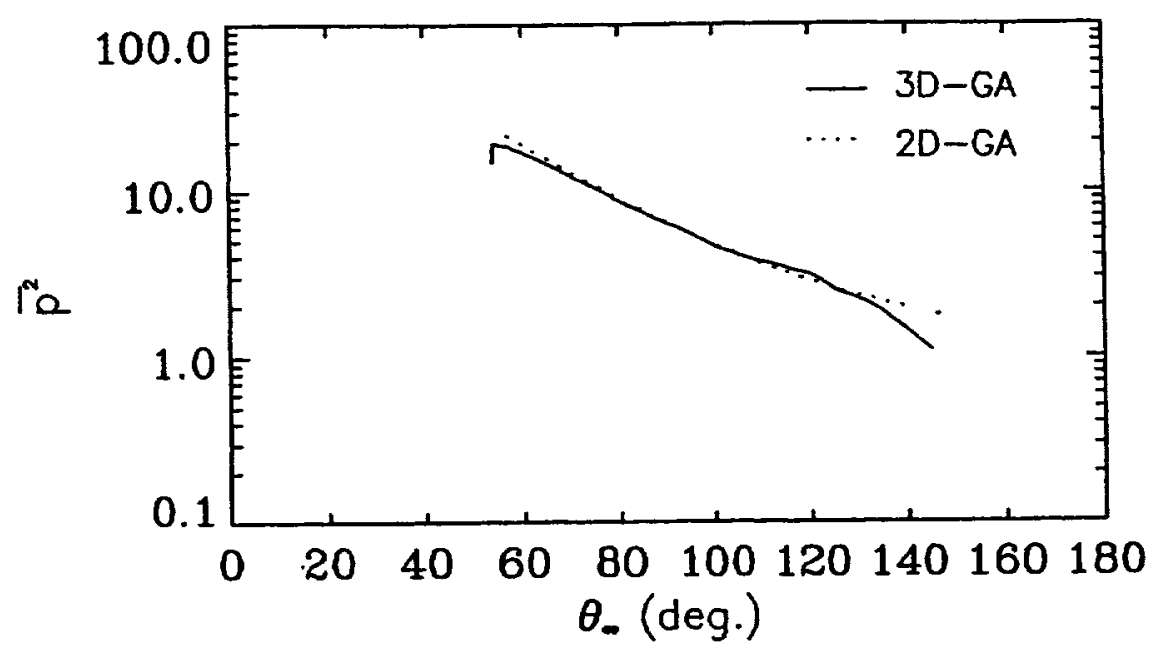

Figure 9.-Noise directivity for a ring source at $X_{0} / D=7$ and $r_{0} / D=0.31$. 


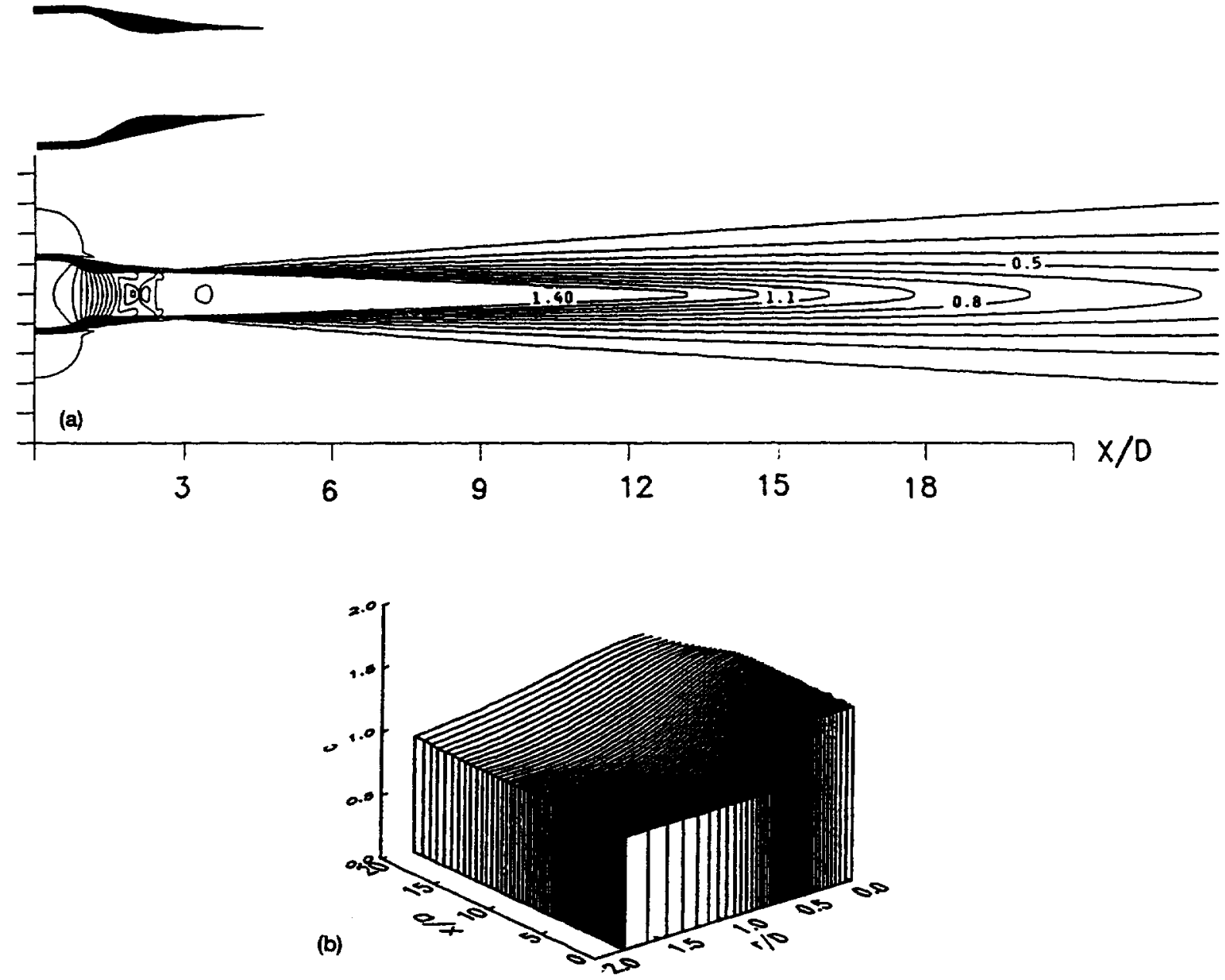

Figure 10.-(a) Mach number contour plot for the comvergent-divergent nozzle at the design Mach number of $M=1.4$. (b) Sound speed of the supersonic jet.

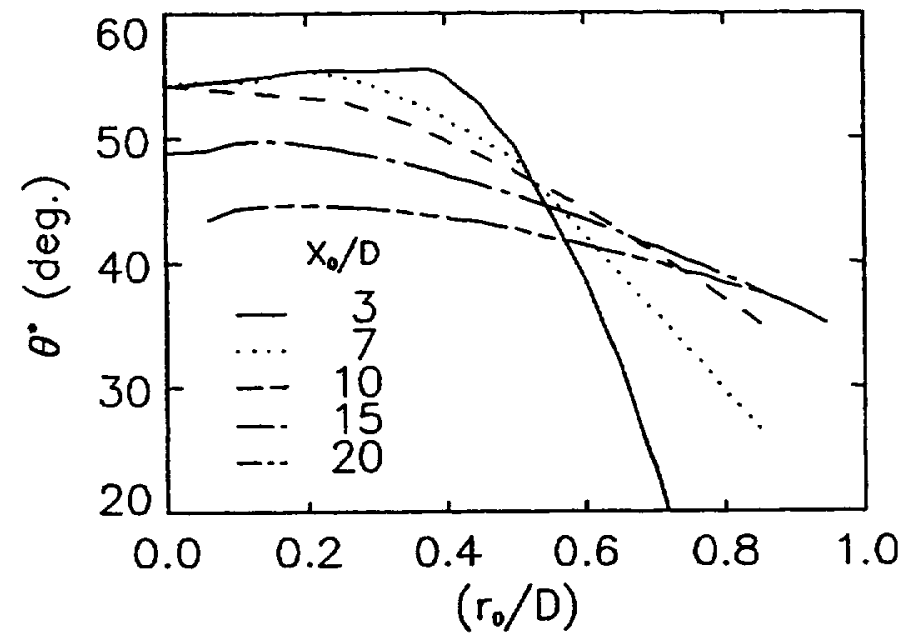

Figure 11.-Boundary of the zone of silence of the supersonic jet. 

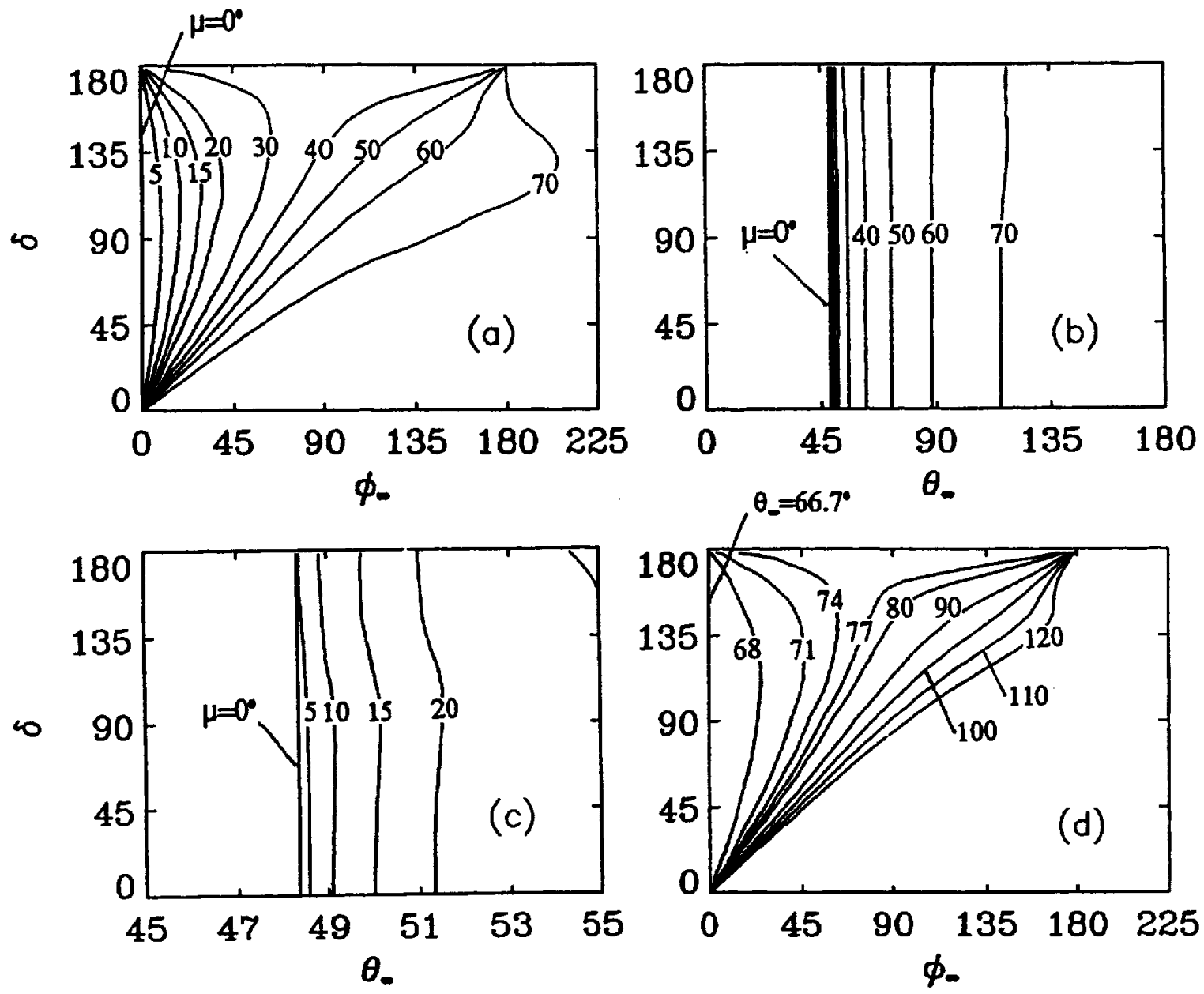

Figure 12.-Directivity angles of the supersonic jet for an off-axis source at $X_{0} / D=7$ and $r_{0} D=0.50$. Figures (a) and (b) are the 3D-GA prectictions. (c) is the enlarged (b). (d) is the 2D-GA prediction. 

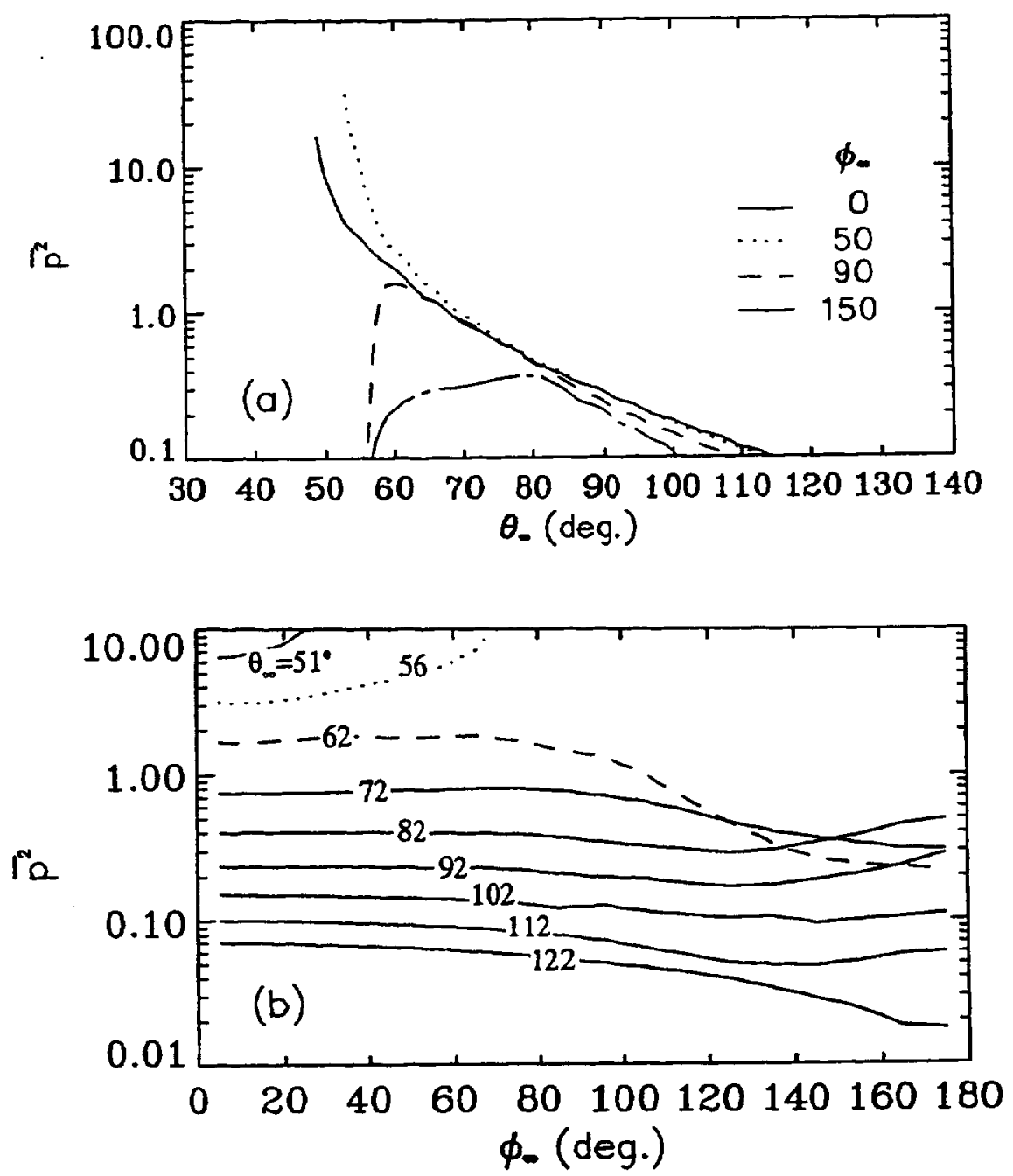

Figure 13.-Noise directivity for an off-axis source within the supersonic jet at $X_{0} / D=7$ and $r_{0} / D=0.50$. (a) Polar directivity. (b) Azimuthal directivity. 

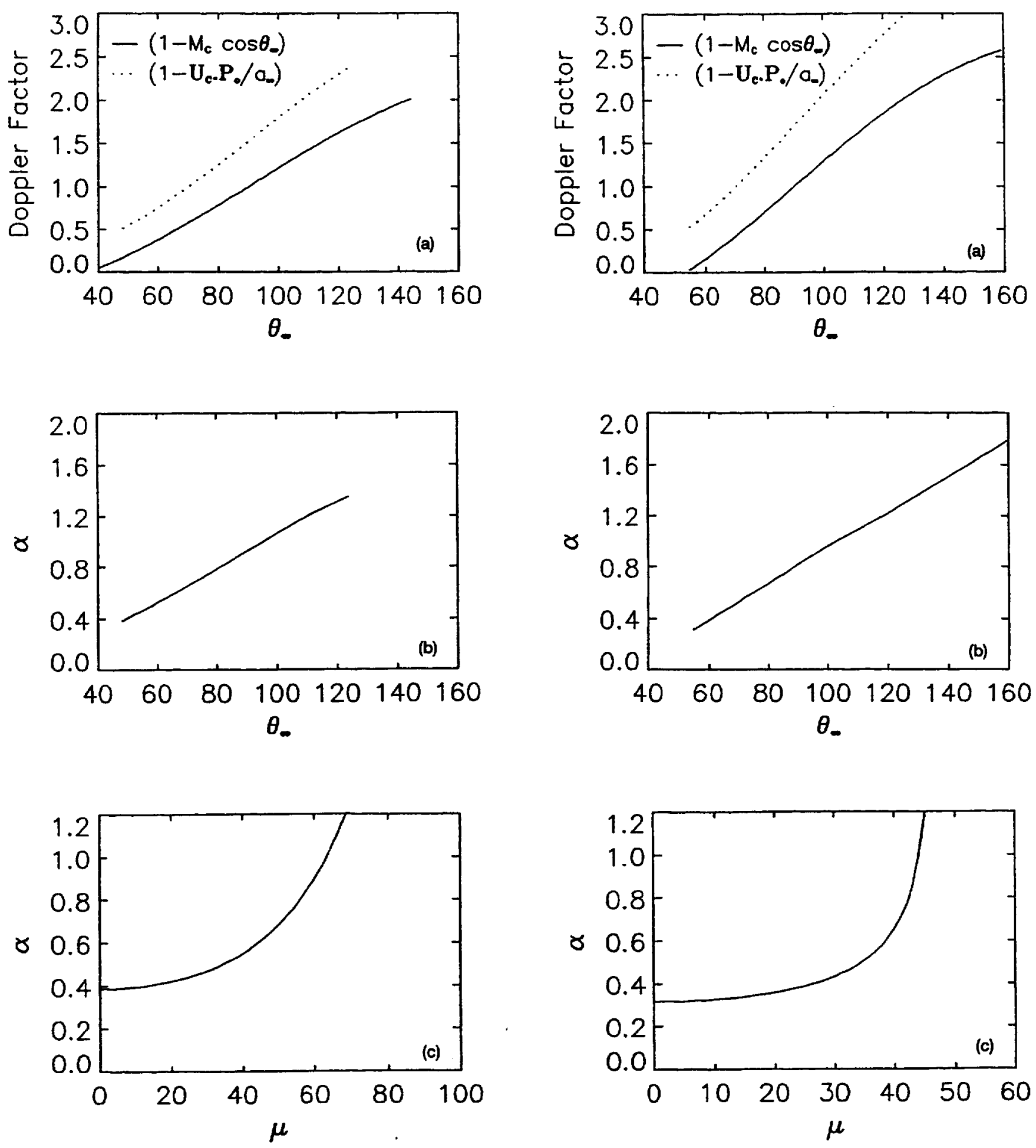

Figure 14.-(a) Doppler factor of the supersonic jet for the source of figure 13. (b) Empirical constant of the modified Doppler factor for the source of figure 14a. (c) Empirical constant of the modified Doppler factor for the source of figure 142

Figure 15.- (a) Doppler factor of the supersonic jet with the source point at $X_{0} / D=7$ and $r_{0} / D=0.25$. (b) Empirical constant of the modified Doppler factor for the source of figure 15a (c) Empirical constant of the modified Doppler factor for the source of figure $15 a$ 


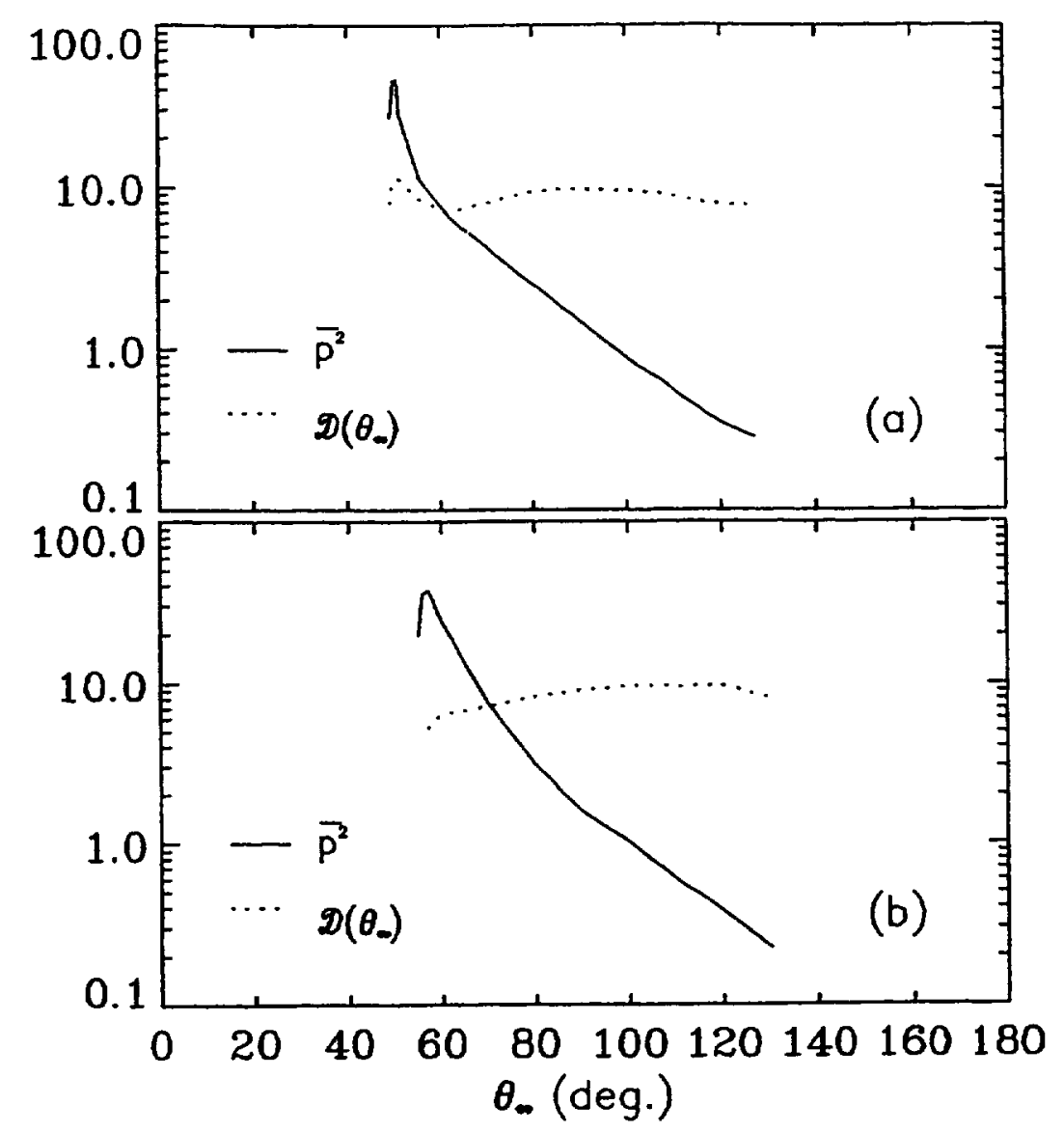

Figure 16.-Noise directivity for a ring source within the supersonic jet at $X_{0} / D=7$. (a) $r_{d} / D=0.50$. (b) $r_{d} / D=0.25$.

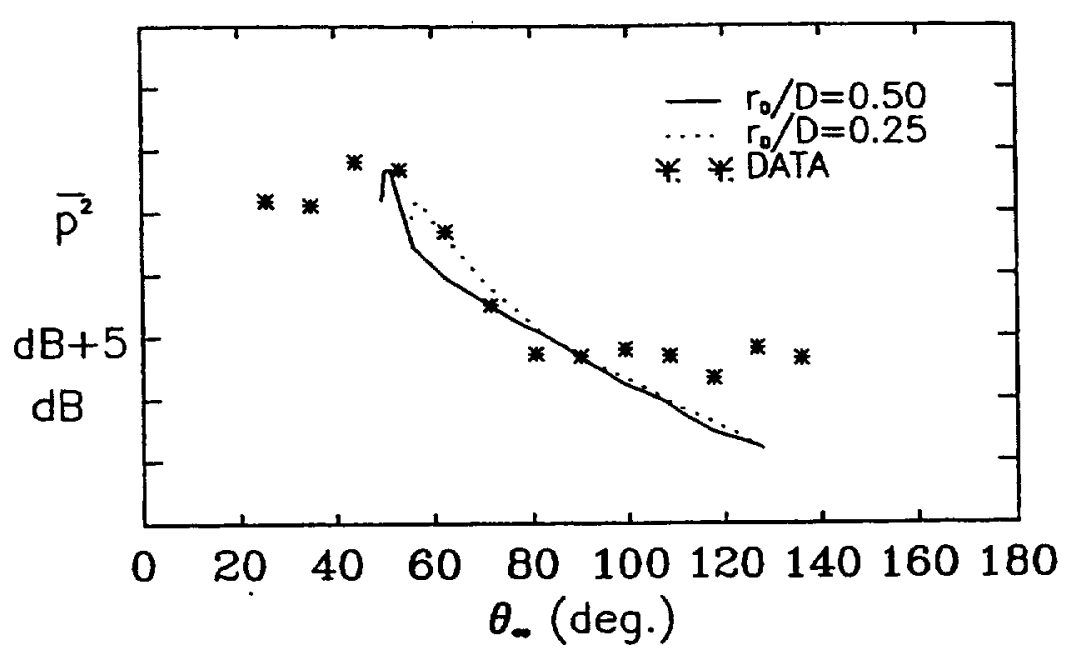

Figure 17.-Comparison between data and high frequency approximation. Predictions are for a ring source at $X_{0} / D=7$. 


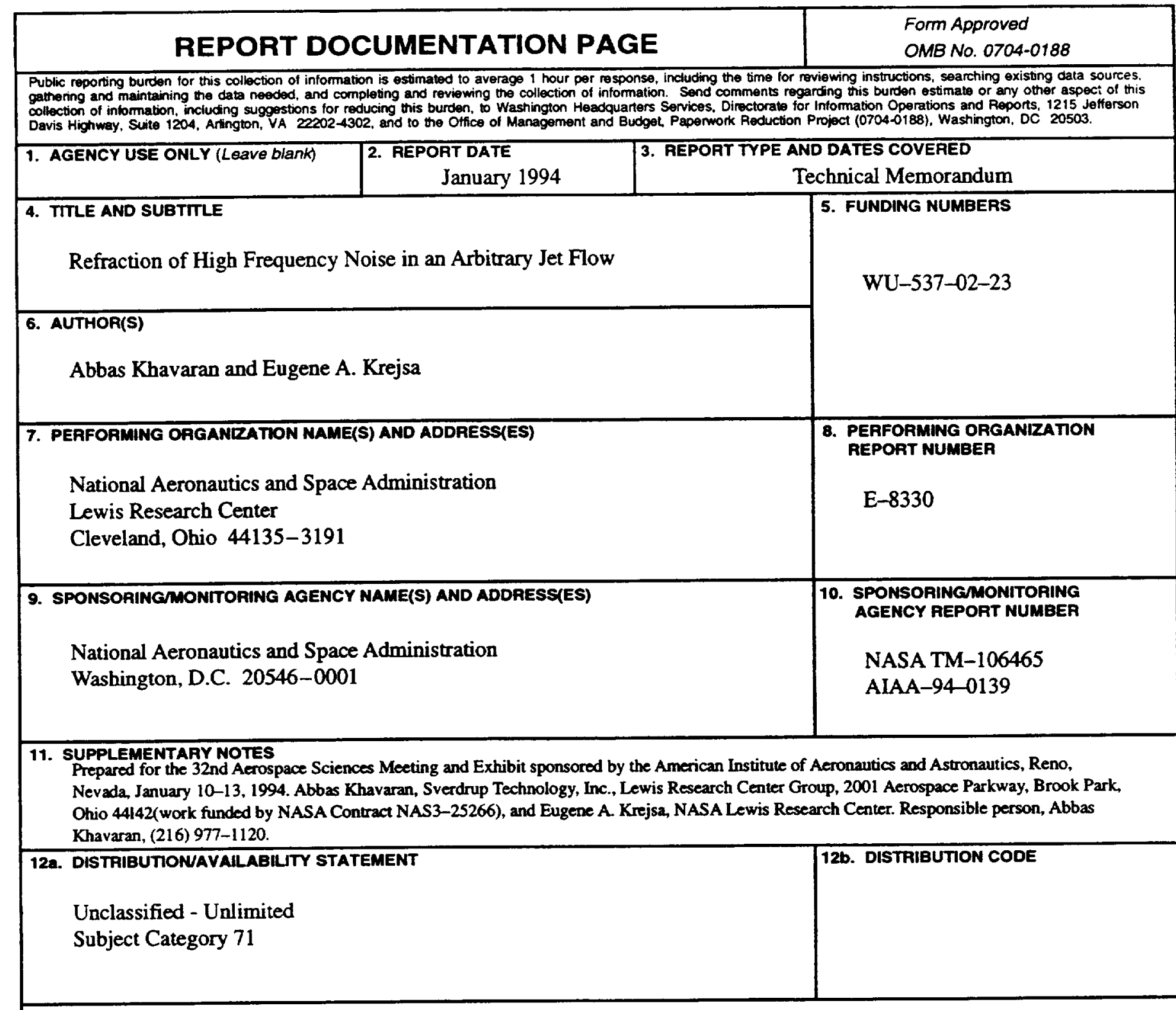

13. ABSTRACT (Maximum 200 words)

Refraction of high frequency noise by mean flow gradients in a jet is studied using the ray-tracing methods of geometrical acoustics. Both the two-dimensional(2D) and three-dimensional (3D) formulations are considered. In the former case, the mean flow is assumed parallel and the governing propagation equations are described by a system of four first order ordinary differential equations. The 3D formulation, on the other hand, accounts for the jet spreading as well as the axial flow development. In this case a system of six first order differential equations are solved to trace a ray from its source location to an observer in the far field. For subsonic jets with a small spreading angle both methods lead to similar results outside the zone of silence. However, with increasing jet speed the two prediction models diverge to the point where the parallel flow assumption is no longer justified. The Doppler factor of supersonic jets as influenced by the refraction effects is discussed and compared with the conventional modified Doppler factor.

\section{SUBJECT TERMS}

Jet noise; Acoustics; Aero-dynamic noise; Supersonic jet noise

\begin{tabular}{|c|c|}
\hline 17. SECURITY CLASSIFICATION & $\begin{array}{c}\text { 18. SECURITY CLASSIFICATION } \\
\text { OF REPORT } \\
\text { OF THIS PAGE } \\
\text { Unclassified }\end{array}$ \\
Unclassified
\end{tabular}

19. SECURTY CLASSIFICATION OF ABSTRACT Unclassified 